\title{
Sexually Dimorphic Properties in Meiotic Chromosome
}

\author{
Kei-Ichiro Ishiguro \\ Department of Chromosome Biology, Institute of Molecular Embryology and Genetics (IMEG), Kumamoto \\ University, Kumamoto, Japan
}

\section{Keywords}

Chromosome axis; Cohesin - Crossover · Meiosis .

Synaptonemal complex

\begin{abstract}
Background: Meiosis is a crucial process for germ cell development. It consists of 1 round of DNA replication followed by 2 rounds of chromosome segregation, producing haploid gametes from diploid cells. During meiotic prophase, chromosomes are organized into axis-loop structures, which underlie meiosis-specific events such as meiotic recombination and homolog synapsis. Meiosis-specific cohesin plays a pivotal role in establishing higher-order chromosome architecture and regulating chromosome dynamics. Summary: Notably, sexually dimorphic properties of chromosome architecture are prominent during meiotic prophase, despite the same axial proteins being conserved between male and female. The difference in chromosome structure between the sexes gives sexual differences in the regulation of meiotic recombination and crossover distribution. Key Messages: This review mainly focuses on the sexual differences of meiosis from the viewpoint of chromosome structure in mammals, elucidating the differences in meiotic recombination and homolog synapsis between the sexes.
\end{abstract}

(c) 2022 S. Karger AG, Basel

\section{Introduction}

Meiosis is a crucial process for germ cell development, which produces haploid gametes from diploid cells. Upon stimulation by retinoic acid [Bowles et al., 2006; Koubova et al., 2006], STRA 8 and MEIOSIN direct the switching from mitosis to meiosis in embryonic oocytes and in postnatal spermatocytes [Baltus et al., 2006; Anderson et al., 2008; Kojima et al., 2019; Ishiguro et al., 2020]. Meiosis consists of 1 round of DNA replication followed by 2 rounds of chromosome segregation. Meiotic entry is concomitant with pre-meiotic S phase and is followed by meiotic prophase, a prolonged G2 phase, when meiosisspecific chromosomal events sequentially occur. During meiotic prophase, chromosomes are organized into axisloop structures, which provide the structural framework for meiosis-specific events such as meiotic recombination and homolog synapsis [Handel and Schimenti, 2010; Zickler and Kleckner, 2015]. Interestingly, sex-specific differences of chromosome architecture are observed during meiotic prophase, despite the same axial proteins being conserved between the sexes. Accumulating evidence from genetic studies demonstrated that female meiosis is more tolerant to deficiencies in meiotic factors than male meiosis. The robustness of female meiosis is brought at the cost of increased aneuploidy in the resul-

\footnotetext{
Karger
} 


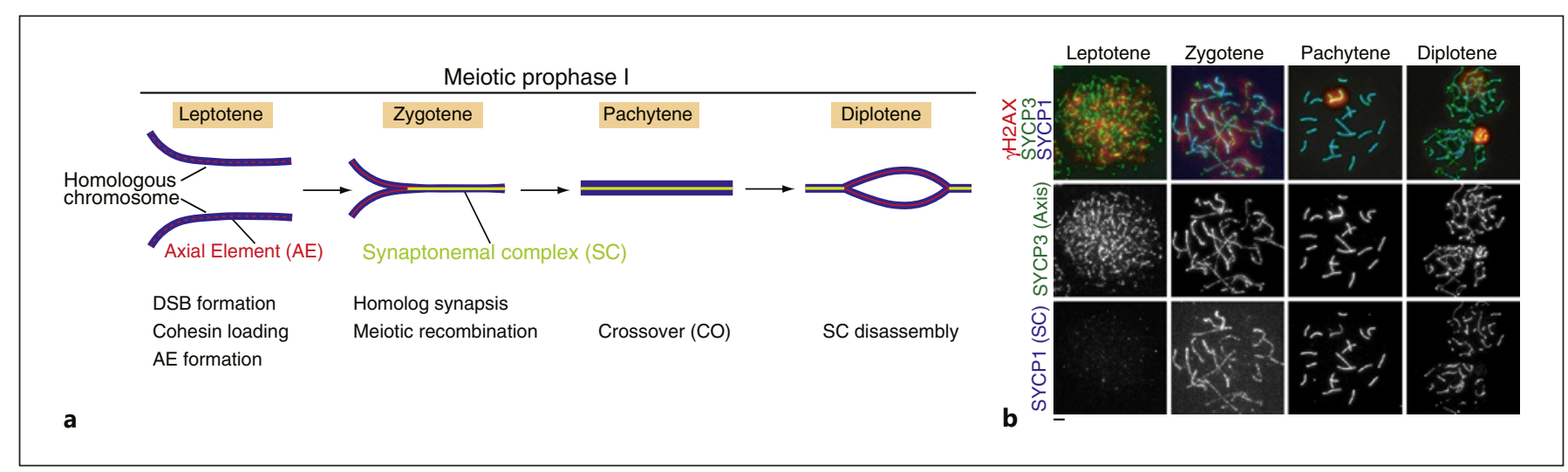

Fig. 1. Schematic of chromosome dynamics during meiosis. a Meiotic prophase I is a prolonged G2 phase and is divided into 4 substages according to the chromosome morphology. During meiotic prophase I, sister chromatids form an axial element (AE). Cohesins load along sister chromatids. Double-strand breaks (DSBs) are generated at leptotene. Homologous chromosomes undergo synapsis through leptotene to zygotene. The synaptonemal complex

tant oocytes [Hunt and Hassold, 2002]. This review focuses on the sexual differences in chromosome structure, meiotic recombination, and homolog synapsis in mammals.

\section{Sexual Differences in Meiotic Chromosome Axis and Synaptonemal Complex}

During meiotic prophase, homologous chromosomes (homologs) undergo pairing/synapsis and meiotic recombination, yielding chiasmata whereby 2 homologs are physically connected [Barzel and Kupiec, 2008; Keeney et al., 2014; Hunter, 2015; Cahoon and Hawley, 2016] (Fig. 1a). During meiotic prophase I, chromosomes are organized into proteinaceous structures, termed axial element (AE) or chromosome axis, from which chromatin loops are protruded. The AE appears at leptotene, which is marked by its main components, SYCP2 [Yang et al., 2006; Fujiwara et al., 2020] and SYCP3 [Yuan et al., 2002], and develops into a continuous linear structure along the sister chromatid axis (Fig. 1b). The AE provides a scaffold to recruit meiotic recombination machineries that promote double-strand break (DSB) introduction and DSB repair [Baudat et al., 2013]. The AE also underlies the structural basis for the assembly of the synaptonemal complex (SC) that mediates tight association of homologous chromosomes and promotes recombination (Fig. 2) [Zickler and Kleckner, 2015; Cahoon and Hawley, 2016].
(SC) is assembled between the homologous chromosomes at pachytene. Meiotic recombination machineries load on the AE to repair DSBs and generate crossovers between homologous chromosomes, yielding physical linkages called chiasmata. At diplotene, the SC is disassembled. b Immunostaining of SYCP1 (a marker of SC), SYCP3 (a marker of SC), and $\gamma \mathrm{H} 2 \mathrm{AX}$ (a marker of DSBs) on spread chromosomes of spermatocytes. Scale bar, $5 \mu \mathrm{m}$.

Notably, although the main components of the AE are conserved between male and female, organization of the AE and the loops shows sexually dimorphic properties in mouse and human [Morelli and Cohen, 2005; Gruhn et al., 2013; Cahoon and Libuda, 2019]. While the AE length is longer in female than in male, the average length of the loops which protrude from the $\mathrm{AE}$ is shorter in female than in male (Fig. 2). Accordingly, the $\mathrm{SC}$ length at pachytene is longer in female than in male since the $\mathrm{AE}$ constitutes the lateral element (LE) of the SC [Tease and Hulten, 2004]. In addition to the difference in SC length, it has been shown that organization of the SC is different between male and female [Agostinho et al., 2018]. The SC has a tripartite ladder-like structure where transverse filaments (TFs) connect the central element (CE) to the LEs of each homolog (Fig. 2). The super-resolution structured illumination microscopy (SIM) analysis demonstrated that the SC width (the distance between the 2 SYCP3-labeled LEs) is narrower in oocytes $(\sim 143 \mathrm{~nm})$ than spermatocytes $(\sim 210$ $\mathrm{nm}$ ) (Fig. 2). Accordingly, the CE width was narrower and the C-termini of the TF component SYCP1 integrated more deeply into the LE in female SCs. Although it is yet to be clarified how morphological differences in the SC contribute to meiotic recombination and homolog synapsis, genetic studies revealed sexually different requirements of the structural components of the SC. It should be noted that sexual dimorphism is more evident in $S y c p 3$ knockout (KO) mice. In Sycp3 KO male, 
Fig. 2. Schematic of sexual differences in the axis-loop organization and synaptonemal complex (SC). Schematic of the SC in male and female. When the SC is assembled between homologous chromosomes, the axial element (AE) is called lateral element (LE). Transverse filaments link 2 LEs. Cohesin locates at the most inner side of the LE. Organization of the AE and the loops is different between the sexes. The length of the $\mathrm{AE}$ is longer in female than in male. Average length of the loops which protrude from the $\mathrm{AE}$ is shorter in female than in male. Accordingly, the length of the $\mathrm{SC}$ at pachytene is longer in female than in male. The width of the SC is narrower in oocytes than in spermatocytes.

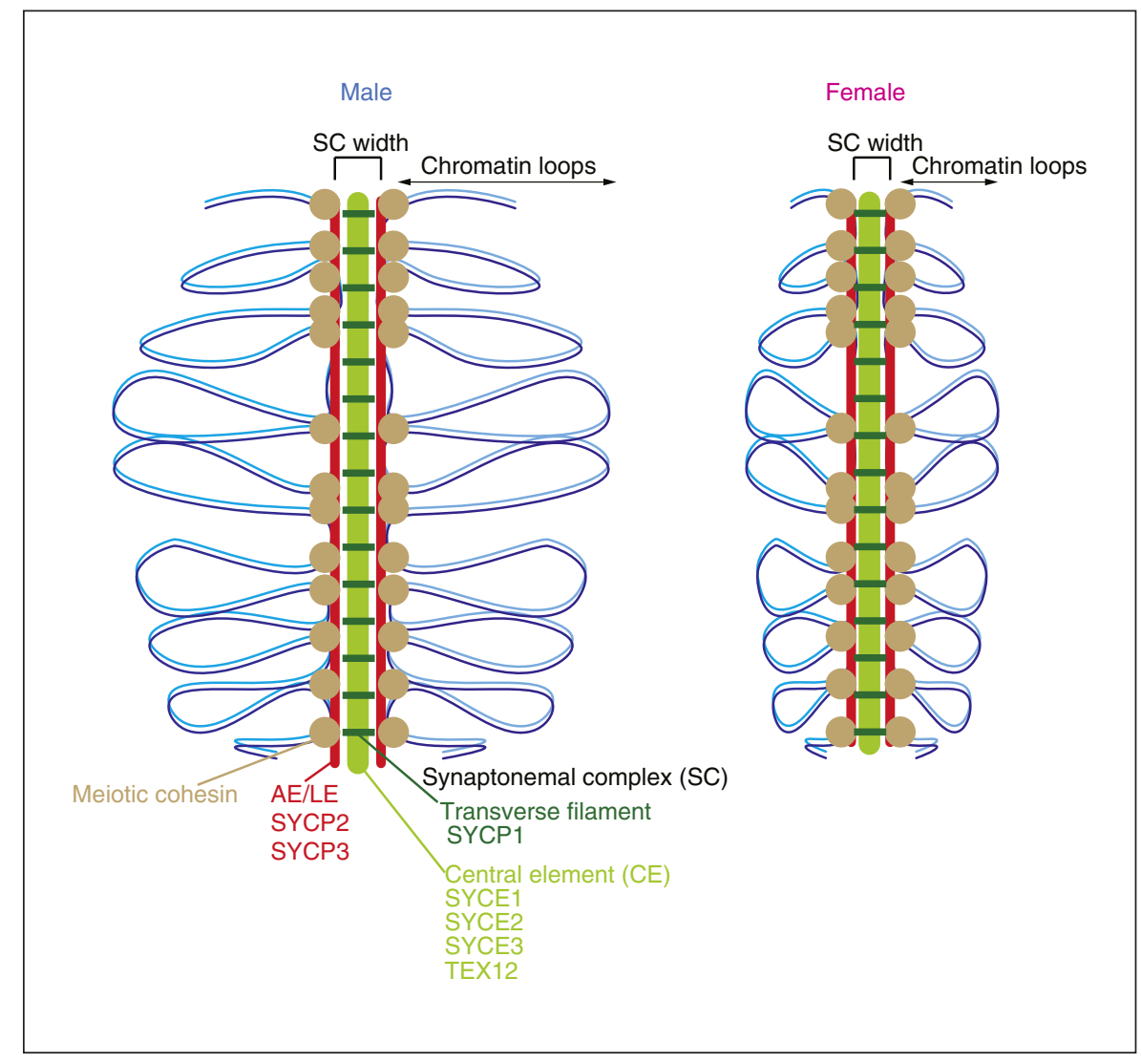

spermatocytes fail to progress beyond the pachytene stage and are eliminated by apoptosis due to defective SC assembly [Yuan et al., 2000]. In contrast, some populations of $S y c p 3 \mathrm{KO}$ oocytes progress through meiotic prophase and reach metaphase I, where some univalent chromosomes lacking chiasmata are observed with severely reduced fertility [Yuan et al., 2002; Kouznetsova et al., 2007]. Thus, the lack of SYCP3 for SC assembly is more tolerated in female than in male meiosis, which may be related to the less active checkpoint response in oocytes. For another example, it has been shown that FKBP6 which is known to be dispensable in female but essential in male, although its exact function is unclear, localizes along the SC [Crackower et al., 2003]. As described later, the $\mathrm{AE}$ and the SC play a pivotal role in recruiting meiotic recombination machineries, but it remains largely elusive how sex-specific differences in the AE/SC structure, such as chromosome axis-loop organization (Fig. 2), contribute to sexually dimorphic properties of meiotic recombination and chromosome dynamics in mammalian meiosis.

Sex-Specific Difference in Meiotic Chromosome

\section{Sexually Different Requirements of Meiosis-Specific Cohesin Complex}

The emergence of SYCP2 and SYCP3 in the nucleus is preceded by the loading of cohesin on the chromatins during the pre-meiotic S phase or early leptotene. Notably, the cohesin complex in meiosis differs from that in mitosis [Ishiguro, 2019]. In mammalian germ cells, there are 2 types of meiosis-specific cohesin complexes, one that contains REC8 [Eijpe et al., 2003; Lee et al., 2003] and another that contains RAD21L [Ishiguro et al., 2011; Lee and Hirano, 2011] (Fig. 3a).

In meiosis, the cohesin complex plays crucial roles not only in sister chromatid cohesion but also in numerous meiosis-specific chromosomal dynamics such as homolog pairing/synapsis and meiotic recombination. The "cohesin axial core" is pre-formed between sister chromatids, which subsequently acts as a framework for the formation of the AE (Fig. 3b). The "cohesin axial core" is formed even in the absence of a major AE component SYCP2 [Fujiwara et al., 2020] or SYCP3 [Pelttari et al., 2001; Fujiwara et al., 2020] (Fig. 3c). 


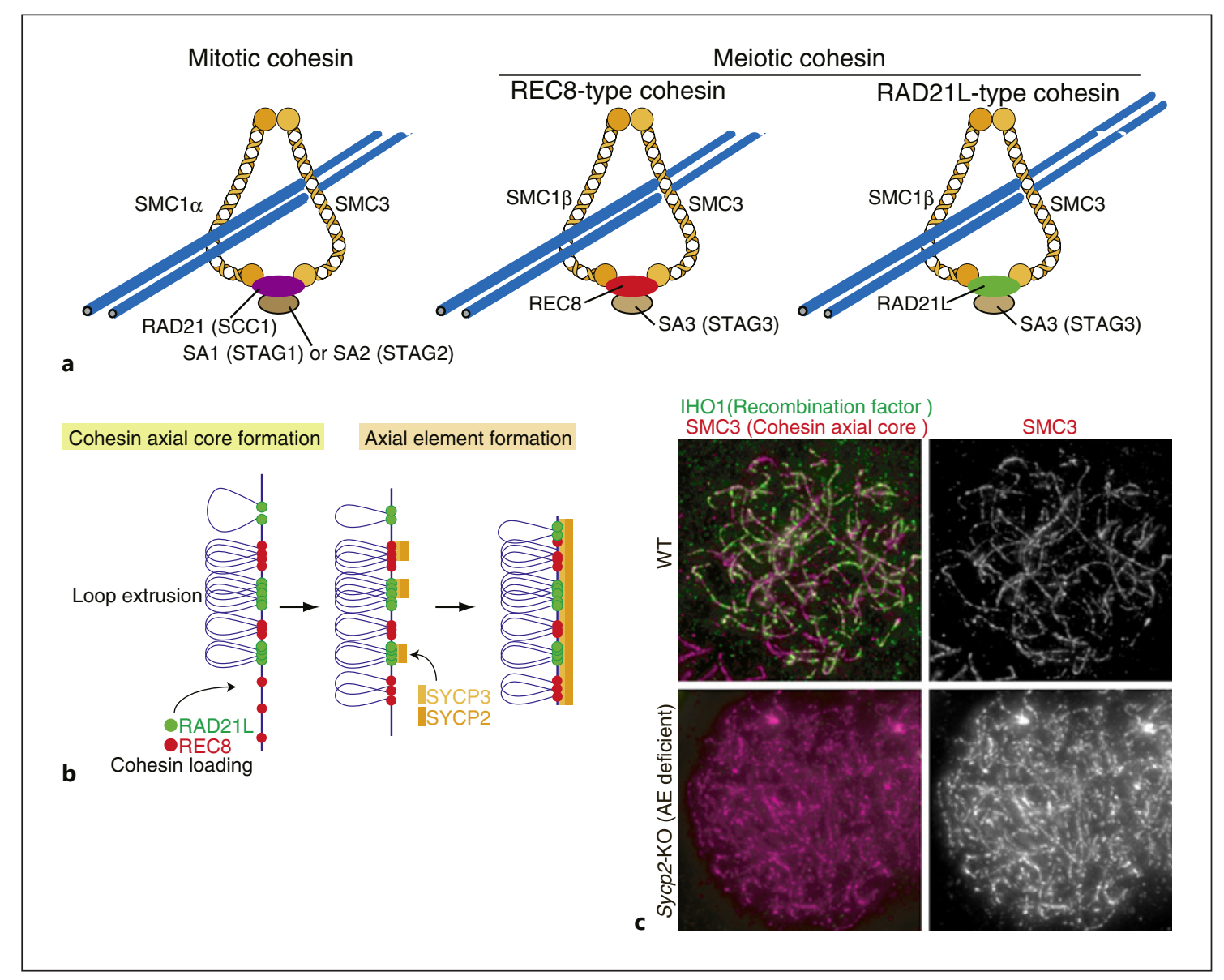

Fig. 3. The meiosis-specific cohesin underlies chromosome axis. a Sister chromatids are held together by cohesin complexes in mitosis and meiosis. The cohesin complex consists of 4 core subunits, and the composition differs between meiosis and mitosis. In mitosis, it consists of SMC1 1 , SMC3, the kleisin family protein RAD21 (also known as SCC1), and SA1 (also known as STAG1) or SA2 (also known as STAG2). In meiosis of mammalian germ cells, it consists of SMC1 $\beta$, SMC3, meiosis-specific kleisin subunits REC8 or RAD21L, and SA3 (also known as STAG3). b Schematic model of REC8 and RAD21L loaded on the chromatin during meiotic prophase. Chromatins are shown in blue. REC8- and RAD21Lcohesins are shown in red and green circles, respectively. AE com-

Disruption of either REC8 [Bannister et al., 2004; Xu et al., 2005] or RAD21L [Herran et al., 2011; Ishiguro et al., 2014] leads to impaired formation of the AE. In Rec8 $\mathrm{KO}$ spermatocytes, the length of the AE is shorter compared to wild type. In Rad21L KO spermatocytes, AE becomes discontinuous and fragmented. These results indicate that RAD21L- and REC8-cohesin complexes differently contribute to the formation of the AE. As shown by mostly abolished formation of $\mathrm{AE}$ in $\operatorname{Rec} 8 / \mathrm{Rad} 21 \mathrm{~L}$ double $\mathrm{KO}$ [Llano et al., 2012] [Ishiguro et al., 2014], the meiotic ponents SYCP2 and SYCP3 are shown in orange and light orange rectangles, respectively. In meiotic prophase, REC8- and RAD21Lcohesins localize along the chromosomes before or during premeiotic DNA replication (preleptotene) and leptotene, and persist throughout the first meiotic division. REC8 and RAD21L show mutually exclusive localization along the chromosomes. The "cohesin axial core" is pre-formed along the chromosomes and subsequently acts as a framework for the formation of AE. c The "cohesin axial core" is formed even in the absence of a major AE component SYCP2. Meiotic recombination factor (IHO1) shows foci along the AE in WT spermatocyte. AE, axial element.

"cohesin axial core" assembled by REC8- and RAD21Ltype cohesin complexes plays an essential role in AE formation.

It was shown that the components of the AE (SYCP2 and SYCP3) and meiotic cohesin complexes coordinate the formation of the axis-loop [Novak et al., 2008]. In the absence of SYCP3, the length of "cohesin axial core" is extended with reciprocal shortening of the chromatin loop. This suggests that the "cohesin core axis" is longitudinally compacted by the AE component (SYCP3). In the 
Fig. 4. The BRME1-MEILB2 complex plays a role in meiotic recombination. a Immunostaining of BRME1, MEILB2, and SYCP3 (a marker of SC) on spread chromosomes of spermatocytes. BRME1 and MEILB2 colocalize well on the DSB sites. b Immunostaining of DMC1 and SYCP3 on spread chromosomes of spermatocytes. Scale bars, $5 \mu \mathrm{m}$. In Brme1 KO spermatocytes, the loading of DMC1 was decreased. c Schematic showing the processes of homologous recombination during meiotic prophase for WT (male) (left). In Brme1 $\mathrm{KO}$, localization of MEILB2 onto DSBs are reduced (right). Redundant mechanisms that compensate the function of MEILB2 and BRME1 may exist in female, allowing less localization of the recombinases. Red and dark blue lines indicate dsDNAs from homologous chromosomes. Blue circle: BRME1, orange oval: MEILB2, grey circle: ssDNA-binding proteins, green oval: BRCA2, purple oval: RAD51 or DMC1. Schematic was modified and adopted from our previous study [Takemoto et al., 2020].

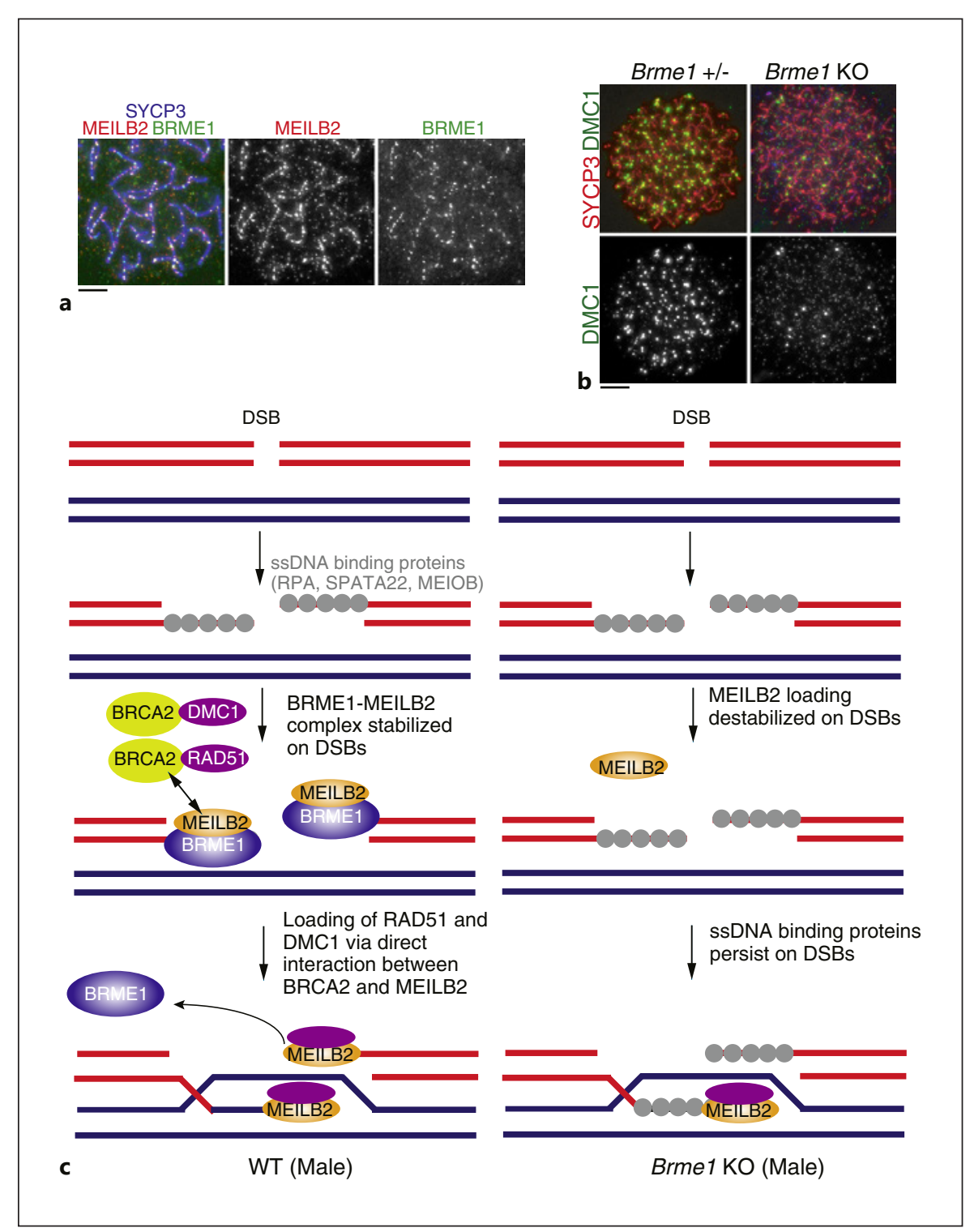

absence of SMC1 $\beta$, a subunit specific to meiotic cohesin complexes (Fig. 3a), the "cohesin axial core" is shortened with reciprocal extension of the chromatin loop size. This suggests that the meiotic cohesin complexes on the chromosome axis act for the formation of the loops. In Smc1//Sycp3 double KOs, the length of the meiotic "cohesin axial core" is restored to the levels comparable to wild type, further suggesting that the AE components act in longitudinal axial compaction of the "cohesin axial core". Thus, the balance between meiotic cohesins and $\mathrm{AE}$ components determines the architecture of the AEchromatin loop during meiotic prophase. It is possible that more loading of cohesin on the chromosomes gives rise to longer "cohesin axial core" in oocytes, which in turn gives longer AE formation in female than in male.

RAD21L- and REC8-cohesin complexes play distinct roles in homolog pairing/synapsis during meiosis [Ishiguro, 2019]. Both Rad21L KO and Rec 8 KO spermatocytes are arrested at zygotene stage with aberrant recombination and SC formation, but the outcomes are different between the 2 KOs. Both spermatocytes show an accumulation of DMC1 and RAD51 foci, suggesting that the recombination process is initiated but the DSB repair process is impaired. In $\operatorname{Rec} 8 \mathrm{KO}$ spermatocytes, the SC assembly occurs between sister chromatids but not between homologs. In the Rad21L KO spermatocytes, the SC is 
aberrantly assembled even between non-homologous chromosomes. Notably, sexual dimorphism is observed in Rad21L KO mice [Herran et al., 2011; Ishiguro et al., 2014]. In Rad21L KO mice, spermatocytes fail to progress beyond pachytene stage without any crossover (CO) recombination and consequently are eliminated by apoptosis. In contrast, Rad21L KO oocytes reach a pachytenelike stage where most of the homologs are successfully synapsed and CO recombination is partially achieved. Thus, lack of RAD21L is tolerated in oocytes for homolog synapsis compared to spermatocytes. This could be because the role of RAD21L-type cohesin is partly substituted by REC8-type cohesin, or it plays less critical roles in $\mathrm{AE}$ formation and homolog interaction in female meiotic prophase. Similar sexual dimorphism is also observed in Smc1 $\beta$ KO [Revenkova et al., 2004] where spermatocytes are arrested at pachytene stage, while oocytes are able to pass through meiotic prophase and reach metaphase I. SMC1 $\alpha$-containing mitotic cohesin complex may partly compensate the role of SMC1 $\beta$-containing cohesin in female meiotic prophase but not in male. Thus, the contribution of meiotic cohesins to AE formation, homolog synapsis, and $\mathrm{CO}$ recombination is differently weighted between male and female meiotic prophase.

\section{Sexual Dimorphism in the Regulation of Meiotic Homologous Recombination}

Meiotic recombination is initiated by the introduction of DSB [Baudat et al., 2013; Lam and Keeney, 2014] by SPO11 [Baudat et al., 2000; Romanienko and CameriniOtero, 2000] and TOPO6BL [Robert et al., 2016; Vrielynck et al., 2016] and is completed by subsequent homologous recombination-mediated repair using homologs instead of sister chromatids as templates [Neale and Keeney, 2006; Handel and Schimenti, 2010; Baudat et al., 2013]. DNA ends at the breaks are resected to form 3 '-extended single-stranded DNA (ssDNA) for the invasion into the homologous template. In meiotic prophase, ssDNA is coated by multiple ssDNA-binding proteins (RPA, MEIOB, SPATA22) to prevent degradation and secondary structure formation [Wold et al., 1998; La Salle et al., 2012; Luo et al., 2013; Souquet et al., 2013; Xu et al., 2017]. Subsequently, RAD51 and DMC1 recombinases promote the removal of ssDNA-binding proteins from the ssDNA and facilitate the invasion of the $3^{\prime}$-extended strand into the duplex of the homolog (Fig. 4c) [Shinohara and Shinohara, 2004; Cloud et al., 2012]. Since the $\mathrm{AE}$ and the SC play a pivotal role in recruiting the factors for meiotic recombination, it is possible that sexually different chromatin architectures affect the processes of meiotic recombination.

In humans, a higher number of RAD51 foci is observed in oocytes than in spermatocytes, suggesting that a higher number of recombination-intermediates is generated in females [Gruhn et al., 2013]. Interestingly, recent studies suggest sexually different mechanisms for the recruitment of RAD51 and DMC1 recombinases onto the ssDNA. It has been shown that meiosis-specific factors MEILB2 (HSF2BP) [Brandsma et al., 2019; Zhang et al., 2019] and BRME1 (C19ORF57) [Felipe-Medina et al., 2020; Shang et al., 2020; Takemoto et al., 2020; Zhang et al., 2020] together with BRCA2 form a ternary complex and promote the loading of RAD51 and DMC1 recombinases onto the DSB sites (Fig. 4a). This process is mediated by at least 3 layers of protein-protein interactions: between BRCA2 and the MEILB2-BRME1 complex, between BRCA2 and RAD51, and between the ssDNAbinding proteins and the MEILB2-BRME1 complex. In Brme1 KO or Meilb2 KO spermatocytes, localization of ssDNA-binding proteins (RPA, MEIOB, SPATA22) was elevated, and reciprocally the loading of RAD51 and DMC1 was decreased (Fig. 4b). This suggests that the removal of the ssDNA-binding proteins was delayed in the absence of the MEILB2-BRME1 complex. Consequently, Brme1 KO and Meilb2 KO mice show severe defects in homolog synapsis and meiotic recombination, leading to impaired male fertility. Thus, in spermatocytes, the MEILB2-BRME1 complex may initially bind to the ssDNAbinding proteins at the resected DNA ends and then facilitate the recruitment of the BRCA2-RAD51 complexes onto the DSB sites by replacing the ssDNA-binding proteins with RAD51 (Fig. 4c).

Notably, although both MEILB2 and BRME1 are expressed in oocytes, they are dispensable for female fertility. Thus, the requirement of MEILB2 and BRME1 for recruiting the recombinases is less strict in females. One possibility is that the checkpoint response may be less active in oocytes, which tolerates the absence of MEILB2 and BRME1. Alternatively, redundant mechanisms may exist to compensate the function of MEILB2 and BRME1 in oocytes. In somatic cells, DSS1 interacts with BRCA2 and facilitates RPA-RAD51 exchange on the ssDNA during homologous recombination [Marston et al., 1999; Zhao et al., 2015]. Although it is yet to be examined whether DSS1 or other factors compensate the function of MEILB2 and BRME1 in meiotic recombination in female, such redundant mechanisms may account for why MEILB2 and BRME1 are not necessarily essential for fe-
6 Sex Dev

DOI: $10.1159 / 000520682$
Ishiguro 
male fertility. Taken together, these lines of evidence suggest the difference in meiotic DSB processing between spermatocytes and oocytes.

\section{Sexual Dimorphism in Crossovers}

While excessive numbers of DSBs are initially generated, at least one of those DSBs is repaired as a CO and the other majorities are repaired as non-COs. COs generate bivalent chromosomes during meiotic recombination, whereby 2 homologous chromosomes are physically connected by chiasmata. Although the exact mechanism of how DSBs are assigned to CO-processing or non-CO-processing is still under extensive study, several layers of regulation and various factors involved in this process are now being elucidated [Hunter, 2015].

Notably, sexual dimorphism in COs have been found in humans and mice [Kong et al., 2010; Gruhn et al., 2013; Liu et al., 2014; Bherer et al., 2017]. Pachytene oocytes show increased number of COs that are indicated by MLH1 foci (Fig. 5a), compared to spermatocytes [Gruhn et al., 2013]. Moreover, the distribution of the COs differ between the sexes. Whereas in male, the MLH1 foci are most likely placed at the regions distal to the centromere, in female they are placed at the interstitial region between the centromere and the telomere. Consistently, genetic maps in human and mouse suggest that recombination events are decreased in sub-telomeric regions in females relative to males [Liu et al., 2014; Bherer et al., 2017], indicating distal COs are disfavored in oocyte meiosis. Furthermore, gene variants that encode SC proteins or meiotic recombination factors cause sex-specific changes in the rates and the distribution of the COs in humans [Halldorsson et al., 2019], which underlies the difference in genome-wide $\mathrm{CO}$ recombination levels between male and female.

\section{Sexually Different Epigenetic Status Contributes to Sex-Biased DSB Hotspots}

In humans and mice, most COs occur in limited genomic regions termed DSB hotspots, where DSBs are frequently generated. Notably, recent studies suggest that the increased number of COs in female is not simply due to an increased number of DSBs [de Boer et al., 2015; Brick et al., 2018]. In mice, although more DSBs are generated at the sub-telomeric regions in females than in males, in females COs at distal regions are rather sup-

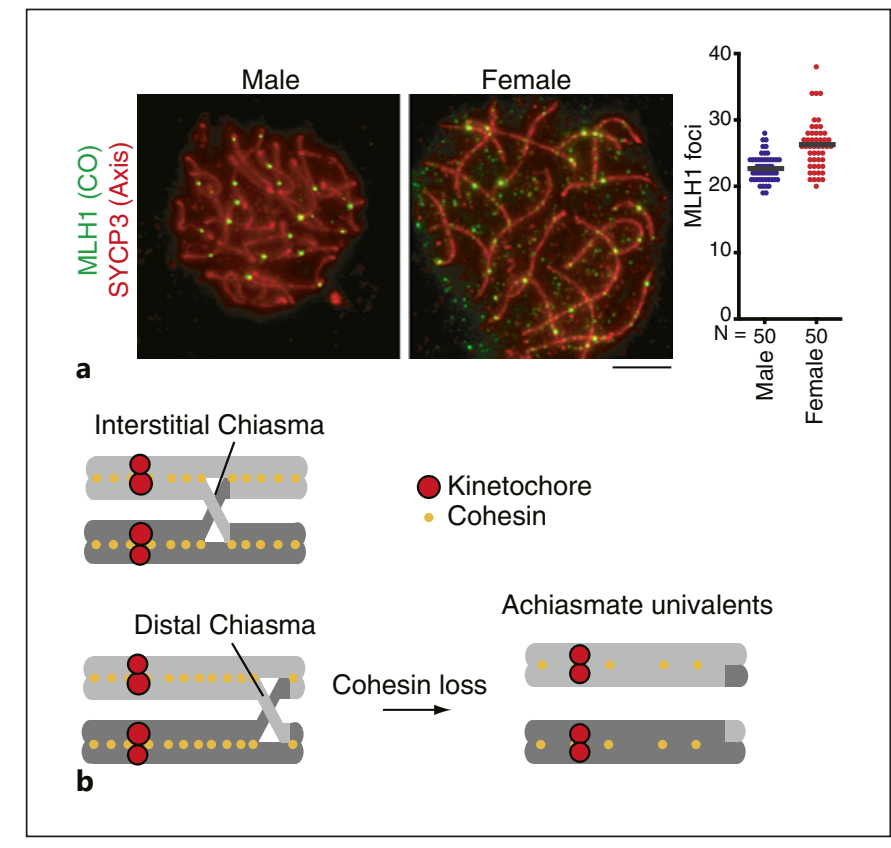

Fig. 5. Crucial role of chiasma position along a chromosome. a Immunostained pachytene spermatocyte ( 8 weeks old) and oocyte (E18.5) are shown. The CO sites are detected by MLH1 foci. CO confers the future position of chiasmata along the chromosomes. Scale bar, $5 \mu \mathrm{m}$. Numbers of MLH1 foci are presented in a scatter plot with medians on the right. The data was adopted from our previous study [Ishiguro et al., 2014]. b Meiotic cohesins (yellow dots) play a role in holding the chiasmata to ensure that 2 homologs are physically connected as bivalents. Distal chiasma raises the risk for producing achiasmate univalent because sister chromatid cohesions are gradually released at the regions distant from the centromere (red circles) during the prophase arrest in oocytes, and the decreased levels of cohesins at the distal arm regions cannot prevent chiasmata from moving and slipping off the chromosome. In addition to the arm cohesion being attenuated with age, the centromeric cohesion also becomes attenuated with age in mice and humans. $\mathrm{CO}$, crossover.

pressed [Brick et al., 2018]. Thus, DSB frequency is not the primary cause of sex-specific differences in distal CO density.

PRDM9 defines most of the DSB hotspots by introducing histone 3 trimethylation at lysine 4 and lysine 36 residues (H3K4me3 and H3K36me3) [Baudat et al., 2013; Diagouraga et al., 2018]. DSBs can be assessed by DMC1ChIP that detects ssDNAs bound to DMC1, which is an early intermediate of meiotic recombination [Brick et al., 2012]. The hotspots defined by DMC1-ChIP-seq correlate with $\mathrm{H} 3 \mathrm{~K} 4 \mathrm{me} 3$ signals. One of the differences between the sexes is that the DMC1-ChIP signal at a hotspot is narrower in females than in males [Brick et al., 2018]. 
The narrower DMC1-ChIP signals in females may reflect shorter DSB ends, DMC1 loading over a shorter distance, or differences in the dynamics of repair between the sexes, which all imply the difference in meiotic DSB processing between the sexes.

Alternatively, another implication of the DMC1-ChIP is that sex biases in recombination is already established before DSB formation. Notably, only a few hotspots are used uniquely in either sex. However, it has been suggested that sex-biased usage of the hotspots contributes to the differences in meiotic recombination between male and female [Brick et al., 2018]. Intriguingly, male-biased, female-biased, and unbiased hotspots defined by DMC1ChIP locate in clusters along the chromosomes. The sexbiased usage of hotspots is partly explained by the different epigenetic status between male and female germ cells. When DSBs are initiated, meiotic chromatins are markedly different between the sexes, showing genome-wide demethylation of the DNA in oocytes but not in spermatocytes [Saitou et al., 2012]. It is well known that DNA methylation alters the site preferences for DNA-binding proteins. Indeed, in males, the PRDM9-binding sites are frequently methylated at male-biased hotspots, while DNA methylation is increased in the region \pm 75 bp adjacent to the PRDM9-binding sites at the female-biased hotspots [Brick et al., 2018]. When the overall DNA methylation level is decreased in male mice with non-functional DNMT3L, the female-biased hotspots become stronger than in the wild type, whereas male-biased hotspots reciprocally get weaker. This suggests that decreasing the DNA methylation level in males leads to an increase in DSBs at female-biased hotspots and reduction at male-biased hotspots. Correspondingly, $\mathrm{H} 3 \mathrm{~K} 4 \mathrm{me} 3$ signals are increased at the female-biased hotspots compared to malebiased hotspots in Dnmt3L mutant male. This indicates that in male, DNA methylation suppresses PRDM9 binding and DSB generation at female-biased hotspots but promotes those events at male-biased hotspots.

Distinct methylation patterns between the sexes modulate sex-specific usage of the hotspot. However, given that DNA is grossly demethylated in oocytes when meiotic recombination is initiated, it is unlikely that DNA methylation solely controls CO distribution in female. Thus, another underlying mechanism for $\mathrm{CO}$ positioning should exist. As mentioned above, sex-biased hotspots cluster along the chromosomes, and sex bias in recombination is already established prior to DSB formation. It is possible that sexual difference in the chromosome architecture itself underlies the sex-biased hotspots during meiosis.

\section{Crucial Role of Chiasma Position for Proper Chromosome Segregation in Oocytes}

What is the physiological meaning of sex-specific difference in $\mathrm{CO}$ distribution? A crucial point regarding $\mathrm{CO}$ distribution is that specific CO sites, together with meiotic cohesins, confer the future position of chiasmata along the chromosomes (Fig. 5b). This is particularly important for stably positioning the chiasma during the long prophase arrest in oocytes. Chiasma plays an essential role in placing the homologous chromosomes in an orientation to face the spindle poles so that they are captured by the microtubules from opposite poles and are segregated evenly during metaphase I [Watanabe, 2012]. At anaphase I, although REC8-cohesins are maintained at the centromere regions until meiosis II, they are cleaved by separase along the chromosome arms, allowing the resolution of the chiasmata and consequently the release of the homologous chromosomes [Buonomo et al., 2000; Kudo et al., 2006]. Then the homologous chromosomes are segregated toward the opposite poles of the spindle with the dissolution of chiasmata. Thus, in meiosis I, homologous chromosomes rather than sister chromatids are segregated into the opposite directions to reduce the chromosome number by half.

It is known that distal chiasma raises the risk for chromosome mis-segregation during meiosis I, as shown by increased incidence of improper chromosome alignment in the homologs due to loss of chiasma. Indeed, this is the case for aged human and mouse oocytes [Liu and Keefe, 2008; Chiang et al., 2010; Hunt and Hassold, 2010; Lister et al., 2010; Nagaoka et al., 2012; Tsutsumi et al., 2014; Sakakibara et al., 2015; Zielinska et al., 2015]. It has been shown that the level of chromosome-bound REC8 is reduced on the chromosome arms in both naturally aged mouse oocytes [Chiang et al., 2010; Lister et al., 2010] and senescence-accelerated mouse oocytes [Liu and Keefe, 2008]. These observations imply that REC8 cohesin has dissociated from the chromosomes during prophase arrest in the aged cells. Also, aged oocytes exhibit a high incidence of distally connected homologous chromosomes [Lister et al., 2010]. This is probably because decreased levels of arm cohesion cannot prevent chiasmata from moving and slipping off the chromosome (Fig. 5b), which leads to a significant reduction in bivalents with chiasmata. Thus, gradual loss of sister chromatid cohesion along the chromosome arm leads to destabilization of the physical linkage between the homologs in aged oocytes. Therefore, distal COs could be a sign of a risk of 
chromosome segregation errors in aged oocytes, resulting in aneuploidy. Maybe suppression of distal COs will be beneficial for oocytes to minimize age-related chromosome segregation errors.

\section{Conclusion}

Sex-specific differences in the regulation of meiotic recombination and chromosome dynamics attribute to different chromosome structures between the sexes. It is well known that the most striking difference between spermatocytes and oocytes is the sensitivity of the checkpoint responses to the errors in DNA repair and homolog synapsis [Morelli and Cohen, 2005]. Aberrations in the components required for AE formation, SC assembly, and meiotic recombination are somewhat more tolerated in oocytes than in spermatocytes during meiosis, although the underlying mechanisms that confer this difference remain poorly understood.

ATR kinase plays a crucial role in meiotic prophase checkpoints in both sexes. ATR phosphorylates H2AX along unsynapsed axes, thereby promoting meiotic silencing of unsynapsed chromosomes (MSUC)/meiotic sex chromosome inactivation (MSCI) [Burgoyne et al., 2009; Turner, 2015]. In spermatocytes, most XY chromosome regions remain unsynapsed due to limited homology and are silenced in the heterochromatic XY body as marked with $\gamma \mathrm{H} 2 \mathrm{AX}$. XY chromosome silencing is essential for the progression through pachytene and passing the pachytene checkpoints in spermatocytes [Royo et al., 2010]. HORMAD1 is required for SC formation and plays a role in controlling the quality surveillance for homolog alignment. HORMAD1 preferentially associates with unsynapsed chromosome axes and recruits ATR to asynapsed chromatin [Wojtasz et al., 2009; Shin et al., 2010, 2013]. Notably, Hormad1 KO mice show apparently opposite phenotypes in male and female. In Hormad 1 KO male, spermatocytes are defective in XY body formation and consequently are eliminated by apoptosis due to the rejection at the pachytene checkpoint [Daniel et al., 2011]. In a Hormad1 KO background, oocytes show rather better survival even when SC is defective, suggesting in the absence of HORMAD1, oocytes may escape from MSUC or persistent ATR activity and otherwise will be eliminated at meiotic prophase checkpoint.

A similar sexual difference is also observed in the response to heat stress [Hirano, Yoshida, and colleagues, pers. commun]. In mammals, the testes are maintained at lower temperature than the body core. Meiotic progres- sion in testes is sensitive to body core temperature, albeit it is not in ovaries that reside in the abdominal cavity.

Besides the sex-specific differences in meiotic prophase, sexual dimorphism in the mode of chromosome dynamics is observed during meiosis I-meiosis II transition [Kim et al., 2015; Tanno et al., 2020]. In male, spermatocytes pass through a transient interphase-like state with chromosome decondensation and reassembly of the nuclear membrane after the completion of meiosis I. In female, chromosomes are persistently condensed without nuclear compartmentalization after the completion of meiosis I until metaphase II. Although this is partly due to different CDK activity at meiosis I-meiosis II transition between the sexes, the underlying mechanism that gives this difference is yet to be clarified. Furthermore, in oocytes, chromosome segregation during meiotic division I occurs in a different manner from that in spermatocytes [Sakakibara et al., 2015; Yoshida et al., 2015; Kyogoku and Kitajima, 2017], which gives the oocytes higher incidence of error-prone meiotic chromosome segregation [Nagaoka et al., 2012].

It still largely remains enigmatic how sex-specific differences in the chromosome axis-loop organization contribute to sexually dimorphic properties of meiotic recombination, homolog synapsis, and check point response in mammalian meiosis. Future studies with comparisons between spermatocytes and oocytes in mutant mice will elucidate the underlying mechanisms that give sex-specific regulation of chromosome dynamics in mammalian meiosis.

\section{Conflict of Interest Statement}

The authors declare no conflicts of interests.

\section{Funding Sources}

This work was supported in part by KAKENHI grants (\#19H05743, \#20H03265, \#20K21504, \#JP 16H06276) from MEXT Japan; Grants from The Sumitomo Foundation; The Naito Foundation, Astellas Foundation for Research on Metabolic Disorders; Daiichi Sankyo Foundation of Life Science; The Uehara Memorial Foundation; The NOVARTIS Foundation (Japan) for the promotion of Science, Takeda Science Foundation. These funding sources had no role in the design of this study and will not have any role in decision to submit results. 


\section{References}

Agostinho A, Kouznetsova A, Hernandez-Hernandez A, Bernhem K, Blom H, Brismar H, et al. Sexual dimorphism in the width of the mouse synaptonemal complex. J Cell Sci. 2018;131: jcs212548.

Anderson EL, Baltus AE, Roepers-Gajadien HL, Hassold TJ, de Rooij DG, van Pelt AM, et al. Stra8 and its inducer, retinoic acid, regulate meiotic initiation in both spermatogenesis and oogenesis in mice. Proc Natl Acad Sci U S A. 2008; 105:14976-80.

Baltus AE, Menke DB, Hu YC, Goodheart ML, Carpenter AE, de Rooij DG, et al. In germ cells of mouse embryonic ovaries, the decision to enter meiosis precedes premeiotic DNA replication. Nat Genet. 2006;38:1430-4.

Bannister LA, Reinholdt LG, Munroe RJ, Schimenti JC. Positional cloning and characterization of mouse mei8, a disrupted allelle of the meiotic cohesin Rec8. Genesis. 2004;40:184-94.

Barzel A, Kupiec M. Finding a match: how do homologous sequences get together for recombination? Nat Rev Genet. 2008;9:27-37.

Baudat F, Manova K, Yuen JP, Jasin M, Keeney S. Chromosome synapsis defects and sexually dimorphic meiotic progression in mice lacking Spo11. Mol Cell. 2000;6:989-98.

Baudat F, Imai Y, de Massy B. Meiotic recombination in mammals: localization and regulation. Nat Rev Genet. 2013;14:794-806.

Bherer C, Campbell CL, Auton A. Refined genetic maps reveal sexual dimorphism in human meiotic recombination at multiple scales. Nat Commun. 2017;8:14994.

Bowles J, Knight D, Smith C, Wilhelm D, Richman J, Mamiya S, et al. Retinoid signaling determines germ cell fate in mice. Science. 2006;312: 596-600.

Brandsma I, Sato K, van Rossum-Fikkert SE, van Vliet N, Sleddens E, Reuter M, et al. HSF2BP Interacts with a Conserved Domain of BRCA2 and Is Required for Mouse Spermatogenesis. Cell Rep. 2019;27:3790-8e7.

Brick K, Smagulova F, Khil P, Camerini-Otero RD, Petukhova GV. Genetic recombination is directed away from functional genomic elements in mice. Nature. 2012;485:642-5.

Brick K, Thibault-Sennett S, Smagulova F, Lam KG, $\mathrm{Pu} \mathrm{Y}$, Pratto F, et al. Extensive sex differences at the initiation of genetic recombination. Nature. 2018;561:338-42.

Buonomo SB, Clyne RK, Fuchs J, Loidl J, Uhlmann F, Nasmyth K. Disjunction of homologous chromosomes in meiosis I depends on proteolytic cleavage of the meiotic cohesin Rec8 by separin. Cell. 2000;103:387-98.

Burgoyne PS, Mahadevaiah SK, Turner JM. The consequences of asynapsis for mammalian meiosis. Nat Rev Genet. 2009;10:207-16.

Cahoon CK, Hawley RS. Regulating the construction and demolition of the synaptonemal complex. Nat Struct Mol Biol. 2016;23:369-77.

Cahoon CK, Libuda DE. Leagues of their own: sexually dimorphic features of meiotic prophase I. Chromosoma. 2019;128:199-214.
Chiang T, Duncan FE, Schindler K, Schultz RM, Lampson MA. Evidence that weakened centromere cohesion is a leading cause of age-related aneuploidy in oocytes. Curr Biol. 2010;20: $1522-8$.

Cloud V, Chan YL, Grubb J, Budke B, Bishop DK. Rad51 is an accessory factor for Dmc1-mediated joint molecule formation during meiosis. Science. 2012;337:1222-5.

Crackower MA, Kolas NK, Noguchi J, Sarao R, Kikuchi K, Kaneko H, et al. Essential role of Fkbp6 in male fertility and homologous chromosome pairing in meiosis. Science. 2003;300:1291-5.

Daniel K, Lange J, Hached K, Fu J, Anastassiadis K, Roig I, et al. Meiotic homologue alignment and its quality surveillance are controlled by mouse HORMAD1. Nat Cell Biol. 2011;13:599-610.

de Boer E, Jasin M, Keeney S. Local and sex-specific biases in crossover vs. noncrossover outcomes at meiotic recombination hot spots in mice. Genes Dev. 2015;29:1721-33.

Diagouraga B, Clément JAJ, Duret L, Kadlec J, de Massy B, Baudat F. PRDM9 Methyltransferase Activity Is Essential for Meiotic DNA DoubleStrand Break Formation at Its Binding Sites. Mol Cell. 2018;69:853-e6.

Eijpe M, Offenberg H, Jessberger R, Revenkova E, Heyting C. Meiotic cohesin REC8 marks the axial elements of rat synaptonemal complexes before cohesins SMC1beta and SMC3. J Cell Biol. 2003; 160:657-70.

Felipe-Medina N, Caburet S, Sanchez-Saez F, Condezo YB, de Rooij DG, Gomez HL, et al. A missense in HSF2BP causing primary ovarian insufficiency affects meiotic recombination by its novel interactor C19ORF57/BRME1. Elife. 2020;9:e56996.

Fujiwara Y, Horisawa-Takada Y, Inoue E, Tani N, Shibuya H, Fujimura S, et al. Meiotic cohesins mediate initial loading of HORMAD1 to the chromosomes and coordinate SC formation during meiotic prophase. PLoS Genet. 2020;16: e1009048.

Gruhn JR, Rubio C, Broman KW, Hunt PA, Hassold T. Cytological studies of human meiosis: sex-specific differences in recombination originate at, or prior to, establishment of doublestrand breaks. PLoS One. 2013;8:e85075.

Halldorsson BV, Palsson G, Stefansson OA, Jonsson $\mathrm{H}$, Hardarson MT, Eggertsson HP, et al. Characterizing mutagenic effects of recombination through a sequence-level genetic map. Science. 2019;363:eaau1043.

Handel MA, Schimenti JC. Genetics of mammalian meiosis: regulation, dynamics and impact on fertility. Nat Rev Genet. 2010;11:124-36.

Herran Y, Gutierrez-Caballero C, Sanchez-Martin M, Hernandez T, Viera A, Barbero JL, et al. The cohesin subunit RAD21L functions in meiotic synapsis and exhibits sexual dimorphism in fertility. EMBO J. 2011;30:3091-105.

Hunt PA, Hassold TJ. Sex matters in meiosis. Science. 2002;296:2181-3.

Hunt P, Hassold T. Female meiosis: coming unglued with age. Curr Biol. 2010;20:R699-702.
Hunter N. Meiotic Recombination: The Essence of Heredity. Cold Spring Harb Perspect Biol. 2015;7:a016618.

Ishiguro K, Kim J, Fujiyama-Nakamura S, Kato S, Watanabe Y. A new meiosis-specific cohesin complex implicated in the cohesin code for homologous pairing. EMBO Rep. 2011;12:267-75.

Ishiguro KI. The cohesin complex in mammalian meiosis. Genes Cells. 2019;24:6-30.

Ishiguro KI, Kim J, Shibuya H, Hernandez-Hernandez A, Suzuki A, Fukagawa T, et al. Meiosisspecific cohesin mediates homolog recognition in mouse spermatocytes. Genes Dev. 2014;28: 594-607.

Ishiguro KI, Matsuura K, Tani N, Takeda N, Usuki $S$, Yamane M, et al. MEIOSIN Directs the Switch from Mitosis to Meiosis in Mammalian Germ Cells. Dev Cell. 2020;52:429-45 e410.

Keeney S, Lange J, Mohibullah N. Self-organization of meiotic recombination initiation: general principles and molecular pathways. Annu Rev Genet. 2014;48:187-214.

Kim J, Ishiguro KI, Nambu A, Akiyoshi B, Yokobayashi S, Kagami A, et al. Meikin is a conserved regulator of meiosis-I-specific kinetochore function. Nature. 2015;517:466-71.

Kojima ML, de Rooij DG, Page DC. Amplification of a broad transcriptional program by a common factor triggers the meiotic cell cycle in mice. Elife. 2019;8:e43738.

Kong A, Thorleifsson G, Gudbjartsson DF, Masson G, Sigurdsson A, Jonasdottir A, et al. Fine-scale recombination rate differences between sexes, populations and individuals. Nature. 2010;467: 1099-103.

Koubova J, Menke DB, Zhou Q, Capel B, Griswold MD, Page DC. Retinoic acid regulates sex-specific timing of meiotic initiation in mice. Proc Natl Acad Sci U S A. 2006;103:2474-9.

Kouznetsova A, Lister L, Nordenskjöld M, Herbert M, Höög C. Bi-orientation of achiasmatic chromosomes in meiosis I oocytes contributes to aneuploidy in mice. Nat Genet. 2007;39:966-8.

Kudo NR, Wassmann K, Anger M, Schuh M, Wirth $\mathrm{KG}, \mathrm{Xu} \mathrm{H}$, et al. Resolution of chiasmata in oocytes requires separase-mediated proteolysis. Cell. 2006;126:135-46.

Kyogoku H, Kitajima TS. Large Cytoplasm Is Linked to the Error-Prone Nature of Oocytes. Dev Cell. 2017;41:287-e4.

La Salle S, Palmer K, O'Brien M, Schimenti JC, Eppig J, Handel MA. Spata22, a novel vertebratespecific gene, is required for meiotic progress in mouse germ cells. Biol Reprod. 2012;86:45.

Lam I, Keeney S. Mechanism and regulation of meiotic recombination initiation. Cold Spring Harb Perspect Biol. 2014;7:a016634.

Lee J, Hirano T. RAD21L, a novel cohesin subunit implicated in linking homologous chromosomes in mammalian meiosis. J Cell Biol. 2011; 192:263-76

Lee J, Iwai T, Yokota T, Yamashita M. Temporally and spatially selective loss of Rec 8 protein from meiotic chromosomes during mammalian meiosis. J Cell Sci. 2003;116:2781-90. 
Lister LM, Kouznetsova A, Hyslop LA, Kalleas D, Pace SL, Barel JC, et al. Age-related meiotic segregation errors in mammalian oocytes are preceded by depletion of cohesin and Sgo2. Curr Biol. 2010;20:1511-21.

Liu EY, Morgan AP, Chesler EJ, Wang W, Churchill GA, Pardo-Manuel de Villena F. High-resolution sex-specific linkage maps of the mouse reveal polarized distribution of crossovers in male germline. Genetics. 2014;197:91-106.

Liu L, Keefe DL. Defective cohesin is associated with age-dependent misaligned chromosomes in oocytes. Reprod Biomed Online. 2008;16: 103-12.

Llano E, Herran Y, Garcia-Tunon I, Gutierrez-Caballero C, de Alava E, Barbero JL, et al. Meiotic cohesin complexes are essential for the formation of the axial element in mice. J Cell Biol. 2012;197:877-85.

Luo M, Yang F, Leu NA, Landaiche J, Handel MA, Benavente $\mathrm{R}$, et al. MEIOB exhibits singlestranded DNA-binding and exonuclease activities and is essential for meiotic recombination. Nat Commun. 2013;4:2788.

Marston NJ, Richards WJ, Hughes D, Bertwistle D, Marshall CJ, Ashworth A. Interaction between the product of the breast cancer susceptibility gene BRCA2 and DSS1, a protein functionally conserved from yeast to mammals. Mol Cell Biol. 1999; 19:4633-42.

Morelli MA, Cohen PE. Not all germ cells are created equal: aspects of sexual dimorphism in mammalian meiosis. Reproduction. 2005;130: 761-81.

Nagaoka SI, Hassold TJ, Hunt PA. Human aneuploidy: mechanisms and new insights into an age-old problem. Nat Rev Genet. 2012;13:493504.

Neale MJ, Keeney S. Clarifying the mechanics of DNA strand exchange in meiotic recombination. Nature. 2006;442:153-8.

Novak I, Wang H, Revenkova E, Jessberger R, Scherthan H, Höög C. Cohesin Smclbeta determines meiotic chromatin axis loop organization. J Cell Biol. 2008;180:83-90.

Pelttari J, Hoja MR, Yuan L, Liu JG, Brundell E, Moens $\mathrm{P}$, et al. A meiotic chromosomal core consisting of cohesin complex proteins recruits DNA recombination proteins and promotes synapsis in the absence of an axial element in mammalian meiotic cells. Mol Cell Biol. 2001; 21:5667-77.

Revenkova E, Eijpe M, Heyting C, Hodges CA, Hunt PA, Liebe B, et al. Cohesin SMC1 beta is required for meiotic chromosome dynamics, sister chromatid cohesion and DNA recombination. Nat Cell Biol. 2004;6:555-62.

Robert T, Nore A, Brun C, Maffre C, Crimi B, Bourbon HM, et al. The TopoVIB-Like protein family is required for meiotic DNA double-strand break formation. Science. 2016;351:943-9.

Romanienko PJ, Camerini-Otero RD. The mouse Spo11 gene is required for meiotic chromosome synapsis. Mol Cell. 2000;6:975-87.
Royo H, Polikiewicz G, Mahadevaiah SK, Prosser H, Mitchell M, Bradley A, et al. Evidence that meiotic sex chromosome inactivation is essential for male fertility. Curr Biol. 2010;20:211723.

Saitou M, Kagiwada S, Kurimoto K. Epigenetic reprogramming in mouse pre-implantation development and primordial germ cells. Development. 2012;139:15-31.

Sakakibara Y, Hashimoto S, Nakaoka Y, Kouznetsova A, Höög C, Kitajima TS. Bivalent separation into univalents precedes age-related meiosis I errors in oocytes. Nat Commun. 2015;6:7550.

Shang Y, Huang T, Liu H, Liu Y, Liang H, Yu X, et al. MEIOK21: a new component of meiotic recombination bridges required for spermatogenesis. Nucleic Acids Res. 2020;48:6624-39.

Shin YH, Choi Y, Erdin SU, Yatsenko SA, Kloc M, Yang F, et al. Hormad1 mutation disrupts synaptonemal complex formation, recombination, and chromosome segregation in mammalian meiosis. PLoS Genet. 2010;6:e1001190.

Shin YH, McGuire MM, Rajkovic A. Mouse HORMAD1 is a meiosis i checkpoint protein that modulates DNA double- strand break repair during female meiosis. Biol Reprod. 2013;89: 29.

Shinohara A, Shinohara M. Roles of RecA homologues Rad51 and Dmc1 during meiotic recombination. Cytogenet Genome Res. 2004;107: 201-7.

Souquet B, Abby E, Herve R, Finsterbusch F, Tourpin S, Le Bouffant R, et al. MEIOB targets single-strand DNA and is necessary for meiotic recombination. PLoS Genet. 2013;9:e1003784.

Takemoto K, Tani N, Takada-Horisawa Y, Fujimura S, Tanno N, Yamane M, et al. Meiosis-Specific C19orf57/4930432K21Rik/BRME1 Modulates Localization of RAD51 and DMC1 to DSBs in Mouse Meiotic Recombination. Cell Rep. 2020;31:107686.

Tanno N, Kuninaka S, Fujimura S, Takemoto K, Okamura K, Takeda N, et al. Phosphorylation of the Anaphase Promoting Complex activator FZR1/CDH1 is required for Meiosis II entry in mouse male germ cell. Sci Rep. 2020;10:10094.

Tease C, Hultén MA. Inter-sex variation in synaptonemal complex lengths largely determine the different recombination rates in male and $\mathrm{fe}$ male germ cells. Cytogenet Genome Res. 2004; 107:208-15.

Tsutsumi M, Fujiwara R, Nishizawa H, Ito M, Kogo $\mathrm{H}$, Inagaki $\mathrm{H}$, et al. Age-related decrease of meiotic cohesins in human oocytes. PLoS One. 2014;9:e96710.

Turner JM. Meiotic Silencing in Mammals. Annu Rev Genet. 2015;49:395-412.

Vrielynck N, Chambon A, Vezon D, Pereira L, Chelysheva L, De Muyt A, et al. A DNA topoisomerase VI-like complex initiates meiotic recombination. Science. 2016;351:939-43.

Watanabe Y. Geometry and force behind kinetochore orientation: lessons from meiosis. Nat Rev Mol Cell Biol. 2012;13:370-82.
Wojtasz L, Daniel K, Roig I, Bolcun-Filas E, Xu H, Boonsanay V, et al. Mouse HORMAD1 and HORMAD2, two conserved meiotic chromosomal proteins, are depleted from synapsed chromosome axes with the help of TRIP13 AAA-ATPase. PLoS Genet. 2009;5:e1000702.

Wold S, Boye E, Slater S, Kleckner N, Skarstad K. Effects of purified SeqA protein on oriC-dependent DNA replication in vitro. EMBO J. 1998; 17:4158-65.

Xu H, Beasley MD, Warren WD, van der Horst GT, McKay MJ. Absence of mouse REC8 cohesin promotes synapsis of sister chromatids in meiosis. Dev Cell. 2005;8:949-61.

Xu Y, Greenberg RA, Schonbrunn E, Wang PJ. Meiosis-specific proteins MEIOB and SPATA22 cooperatively associate with the singlestranded DNA-binding replication protein A complex and DNA double-strand breaks. Biol Reprod. 2017;96:1096-104.

Yang F, De La Fuente R, Leu NA, Baumann C, McLaughlin KJ, Wang PJ. Mouse SYCP2 is required for synaptonemal complex assembly and chromosomal synapsis during male meiosis. J Cell Biol. 2006;173:497-507.

Yoshida S, Kaido M, Kitajima TS. Inherent Instability of Correct Kinetochore-Microtubule Attachments during Meiosis I in Oocytes. Dev Cell. 2015;33:589-602.

Yuan L, Liu JG, Hoja MR, Wilbertz J, Nordqvist K, Höög C. Female germ cell aneuploidy and embryo death in mice lacking the meiosis-specific protein SCP3. Science. 2002;296:1115-8.

Yuan L, Liu JG, Zhao J, Brundell E, Daneholt B, Höög C. The murine SCP3 gene is required for synaptonemal complex assembly, chromosome synapsis, and male fertility. Mol Cell. 2000;5:73-83.

Zhang J, Fujiwara Y, Yamamoto S, Shibuya H. A meiosis-specific BRCA2 binding protein recruits recombinases to DNA double-strand breaks to ensure homologous recombination. Nat Commun. 2019;10:722.

Zhang J, Gurusaran M, Fujiwara Y, Zhang K, Echbarthi M, Vorontsov E, et al. The BRCA2-MEILB2-BRME1 complex governs meiotic recombination and impairs the mitotic BRCA2RAD51 function in cancer cells. Nat Commun. 2020;11:2055.

Zhao W, Vaithiyalingam S, San Filippo J, Maranon DG, Jimenez-Sainz J, Fontenay GV, et al. Promotion of BRCA2-Dependent Homologous Recombination by DSS1 via RPA Targeting and DNA Mimicry. Mol Cell. 2015;59:176-87.

Zickler D, Kleckner N. Recombination, Pairing, and Synapsis of Homologs during Meiosis. Cold Spring Harb Perspect Biol. 2015;7: a016626.

Zielinska AP, Holubcova Z, Blayney M, Elder K, Schuh M. Sister kinetochore splitting and precocious disintegration of bivalents could explain the maternal age effect. Elife. 2015;4: e11389.
Sex-Specific Difference in Meiotic Chromosome
Sex Dev 